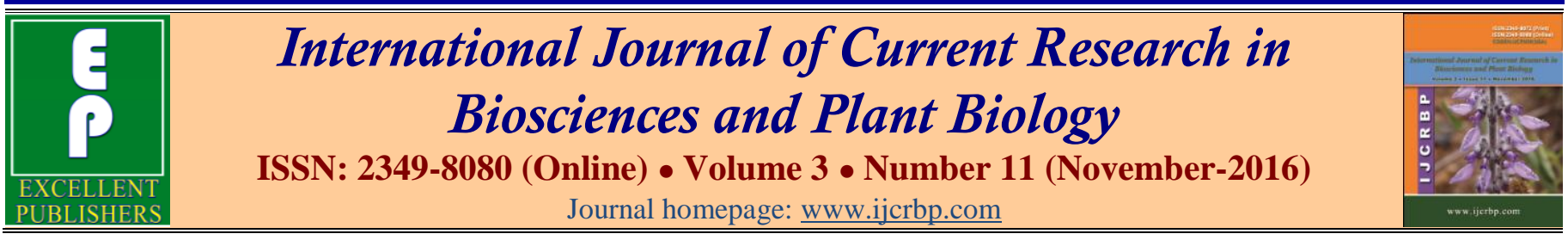

\title{
Cloning and Sequence Analysis of Mevalonate Kinase Gene (CnMVK) from Chamaemelum nobile
}

\author{
Xiangxiang Meng, Weiwei Zhang, Feng Xu*, Jiaping Yan, Xiaomeng Liu, Yong-ling Liao \\ and Jie Chang
}

College of Horticulture and Gardening, Yangtze University, Jingzhou 434025, China

*Corresponding author.

\begin{abstract}
Mevalonate kinase (MVK) is one of the key rate-limiting enzymes in mevalonic (MVA) pathway of terpenoid biosynthesis. To analyse the function of MVK gene in terpenoid biosynthesis in Chamaemelum nobile, the primers were designed according to the transcript unique sequence of $C n M V K$ from the $C$. nobile transcriptome dataset. A MVK gene (designated as CnMVK, GenBank accession number KX894317) was cloned from $C$. nobile using RT-PCR method. The full-length cDNA of CnMVK gene is $1561 \mathrm{bp}$ and contains an open reading frame (ORF) of $1206 \mathrm{bp}$, which encoding a 402 amino-acid protein. The theoretical molecular weight and pI of the CnMVK are $42.8 \mathrm{kDa}$ and 5.79 , respectively. Multi-alignment comparison analysis showed the protein sequence of CnMVK had high similarity with MVK proteins from other plants. Furthermore, CnMVK has similar conserved domains, suggesting CnMVK is one of MVK family mumbers in $C$. nobile. The present study cloned, analyzed the sequence of $C n M V K$ in $C$. nobile will provide a foundation for exploring the molecular mechanism of terpenoid biosynthesis in $C$. nobile.
\end{abstract}

\section{Article Info}

Accepted: 13 October 2016

Available Online: 06 November 2016

\section{Ke y w o r d s}

Chamaemelum nobile

CnMVK gene

Gene cloning

Sequence analysis

\section{Introduction}

Chamaemelum nobile is a herbaceous plant which belongs to Matricaria. The flowers of $C$. nobile rich in volatile aromatic oil, including $\alpha$-bisabolol, $\alpha$-bisabolol Alcohol Oxide A and B, chamazulene and flavonoids, the above components have anti-inflammatory, antibacterial, antioxidant and other effects (Carnat et al., 2004; Lemberkovics et al., 1998; Srivastava et al., 2010). Thus, $C$. nobile is widely used in pharmaceuticals, cosmetics and spices and other fields. However, the flowers of $C$. nobile only contains $1-2 \%$ of the volatile oil (Peña et al., 2006), which cannot meet the market demand. As the incidence of drug-resistant bacteria is increasing, the development of natural antimicrobial resources has become particularly important (Gundersen et al., 2015), thus, it has important significance to improve the content of terpenoids of $C$. nobile by genetic engineering.

Terpenoid is currently known as the largest class of plant secondary metabolites, they play an important role in the process of plant growth and development, and many special terpenoid also play an important function in plant cell signal transduction and stress-resistant physiological processes (Holstein and Hohl, 2004). It has been confirmed that the C5 universal precursor in the biosynthesis of natural terpene compounds is mainly 
derived from mevalonate (MVA) pathway (Dewick, 2002). MVA pathway exists in the cytoplasm, with three acetyl Co A as a raw material through a series of enzymatic reaction to produce isopentenyl pyrophosphate (IPP), part of IPP isomerized to form dimethylallyl pyrophosphate (DMAPP). IPP and DMAPP to form geranyl-pyrophosphate (GPP) (Guo et al., 2012). GPP is combined with one IPP unit to produce farnesyl pyrophosphate (FPP), which is a precursor of sesquiterpene. FPP eventually forms sesquiterpene through heterogeneous, cyclization and complexation. MVA is currently considered to be the main pathway of sesquiterpene biosynthesis. Mevalonic kinase, phosphomevalonate kinase, and mevalonate-5pyrophosphate decarboxylase are of the three consecutive ATP-dependent enzymes present in the enzymatic reaction of the mevalonate pathway, and the mevalonate kinase is the first of three consecutive ATPdependent enzymes (Huang et al., 1999). It is responsible for the transfer of the phosphate group at the ATP $\gamma$ position to the hydroxyl group at position 5 of mevalonate, which forms mevalonate-5-phosphate and is associated with the release of ADP and is one of the ratelimiting enzymes controlling the entire metabolic pathway (Chu and Ding, 2003). Related research (Guo et al., 2012; Huang et al., 2015; Wuyun et al., 2014) shows that the expression of MVK gene may directly or indirectly affect the content of terpenoids in medicinal plants.

The corresponding MVK gene has been cloned from Arabidopsis thaliana (Riou et al., 1994), Salvia miltiorrhiza (Ma et al., 2012), Zea mays (Alexandrov et al., 2009), Catharanthus roseus (Wuyun et al., 2014), Eucommia ulmoides (Simkin et al., 2011) and so on. However, the MVK gene has not been reported in $C$. nobile so far. In view of the fact that MVK is the key rate-limiting enzyme in terpenoids synthesis pathway, the CnMVK gene was cloned by RT-PCR and the bioinformatics analysis of the gene was carried out, and to provide a reliable theoretical basis for revealing the mechanism of CnMVK gene regulation on the biosynthesis of sesquiterpenoid compounds of $C$. nobile.

\section{Materials and methods}

\section{Plant material and reagents}

The leaves of $C$. nobile were collected from botanical garden at Yangtze University, China, and placed in a $-80^{\circ} \mathrm{C}$ freezer immediately. In this experiment, both the primers synthesis and DNA sequencing were completed by Shanghai Sangon Biotechnology Company, in China. MiniBEST Plant RNA Extraction kit, PrimeScript ${ }^{\mathrm{TM}} 1 \mathrm{st}$ Strand cDNA Synthesis Kit, Agarose Gel DNA purification Kit Ver.4.0, dNTP, Taq DNA polymerase RNase, pMD18-T vector were purchased from Takara Company (Dalian, China).

\section{Cloning of CnMVK}

Total RNA was isolated from frozen leaves of $C$. nobile using MiniBEST Plant RNA Extraction kit. The extracted RNA was reverse transcribed into cDNA using PrimeScript ${ }^{\mathrm{TM}} 1$ st Strand cDNA Synthesis Kit. The specific primers as given below were designed based on the MVK unigene sequence of $C$. nobile transcriptome data.

\section{CnMVK-Up (5'-CGGACTGAAGCCTCCAACAAC-3') CnMVK-Dn (5'-CGGCCCTTGAGATGGTCATATACTA-3')}

The MVK gene was amplified by reverse transcriptionpolymerase chain reaction (RT-PCR) method. The amplified products were detected by $1 \%$ gel electrophoresis and purified using Agarose Gel DNA purification Kit Ver.4.0. The purified product was cloned into the pMD18-T vector, then transformed into Escherichia coli DH5a. Positive clones were selected and sent to Shanghai Sangon Biotechnology Company for sequencing.

\section{Bioinformatic analysis}

CnMVK gene sequences were translated into amino acid sequences by using DNAMAN software. The open reading frame (ORF) of the $C n M V K$ gene was predicted by using Vector NTI 11.5. Protein homology searches were performed by using the bioinformatics software on NCBI website (http://www.ncbi.nlm.nih.gov/BLAST/). Multiple sequence alignment was performed with the software Vector NTI11.5 program. Phylogenetic tree of MVK proteins was constructed with neighbor-joining method using Clustal X2.0 and MEGA5 (Larkin et al., 2007).

\section{Results and discussion}

\section{Cloning and sequence analysis of CnMVK}

A pair of specific primers was designed according to the MVK unigene sequence of $C$. nobile transcriptome data, the cDNA sequence of $1561 \mathrm{bp}$ was amplified by RTPCR. The comparison analysis shows that the cDNA sequence is highly homologous to the MVK sequences 
of other plants, it indicated that the cloned cDNA sequence was the MVK gene of $C$. nobile. The cDNA sequence was designated as $C n M V K$ and GenBank accession number was KX894317. The full-length cDNA of $C n M V K$ is $1561 \mathrm{bp}$ and contained a $1206 \mathrm{bp}$ ORF which encoded 402 amino acids (Fig. 1).

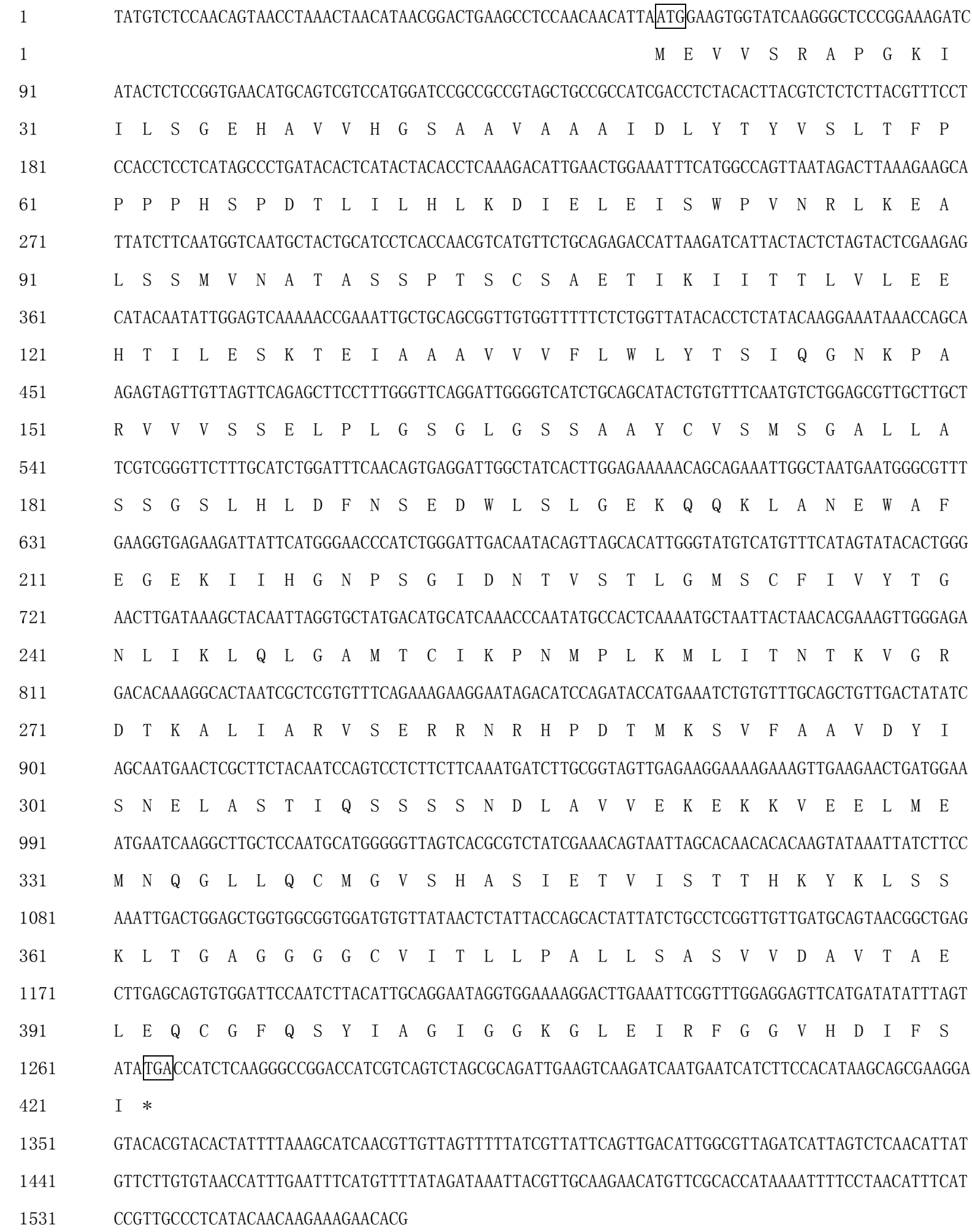

Fig. 1: The Nucleotide sequence and deduced amino acid sequences of the full-length cDNA of CnMVK. The initial codon and the stop codon are highlighted in square box. 


\section{Characterization of CnMVK protein}

ExPASy online (http://web.expasy.org/ compute_pi/) analysis results displayed that the theoretical molecular weight and isoelectric point (pI) of the CnMVK protein were $42.8 \mathrm{kDa}$ and 5.79 , respectively. The multiple sequence alignment of MVK protein sequences among different plants using software Vector NTI11.5 showed that CnMVK is highly homologous to the MVK protein sequences of other plants, further sequencing analysis showed that the protein sequence of CnMVK keep a strong conservation during the molecular evolution
(Fig. 2), different plant MVK genes have similar conserved domains, indicating that each plant MVK gene has a similar enzymatic reaction (Chu and Li, 2003). As shown in Table 1, the protein sequence of CnMVK had high identity with other MVK proteins, it showed 68\%, $67 \%, 67 \%, 66 \%, 66 \%, 66 \%, 65 \%$ and $65 \%$ similarity to MVK proteins from Platycodon grandiflorus, Panax notoginseng, Nicotiana tomentosiformis, Sesamum indicum, Bacopa monnieri, Catharanthus roseus, Solanum pennellii and Olea europaea, respectively, it was further verified that $C n M V K$ is one of members of the MVK gene family of $C$. nobile.

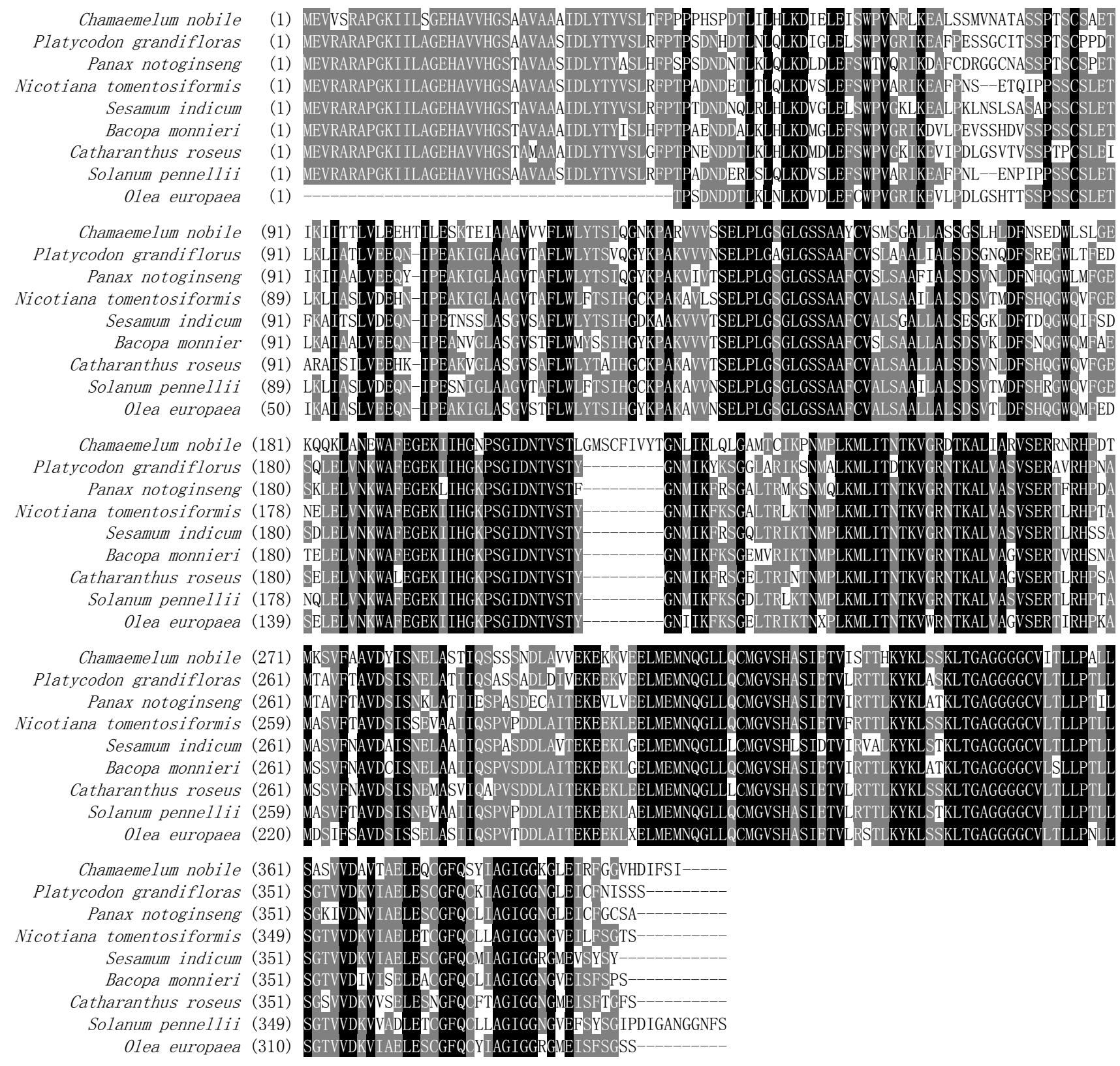

Fig. 2: Multiple sequence alignment of MVK proteins. The completely identical amino acids are indicated with white foreground and black background. 
Table 1. Protein sequence of CnMVK similarity to MVKs of other plant species.

\begin{tabular}{llll}
\hline Species & Accession no. in GenBank & Identity/\% & E-value \\
\hline Platycodon grandiflorus & AGZ15315.1 & 68 & $2 \mathrm{e}-174$ \\
Panax notoginseng & AFN02124.1 & 67 & $1 \mathrm{e}-178$ \\
Nicotiana tomentosiformis & XP_009629945.1 & 67 & $3 \mathrm{e}-172$ \\
Sesamum indicum & XP_011092024.1 & 66 & $1 \mathrm{e}-179$ \\
Bacopa monnieri & AFJ93086.1 & 66 & $6 \mathrm{e}-171$ \\
Catharanthus roseus & ADR65111.1 & 66 & $3 \mathrm{e}-166$ \\
Solanum pennellii & XP_015062317.1 & 65 & 65 \\
Olea europaea & AFS28683.1 & 65 & $3 \mathrm{e}-150$ \\
\hline
\end{tabular}

\section{Phylogenetic analysis of CnMVK}

To investigate the evolutionary relationships among MVK proteins, a phylogenetic tree was constructed based on the deduced amino acid sequences of predicted CnMVK and MVK proteins from other plant species (Fig. 3). MVK phylogenetic tree is divided into two branches of monocotyledons and dicotyledons. C. nobile belonged to dicotyledons in the branch of dicotyledons and monocotyledons. C. nobile has the closest relationship with Cynara cardunculus, it is inferred that CnMVK has similar catalytic activity to MVK protein of $C$. cardunculus. $C$. nobile also has a genetic relationship with other dicotyledons and monocotyledons. This reflects the evolutionary conservation and evolutionary diversity of plant MVK genes, which is consistent with the morphological classification of plants.

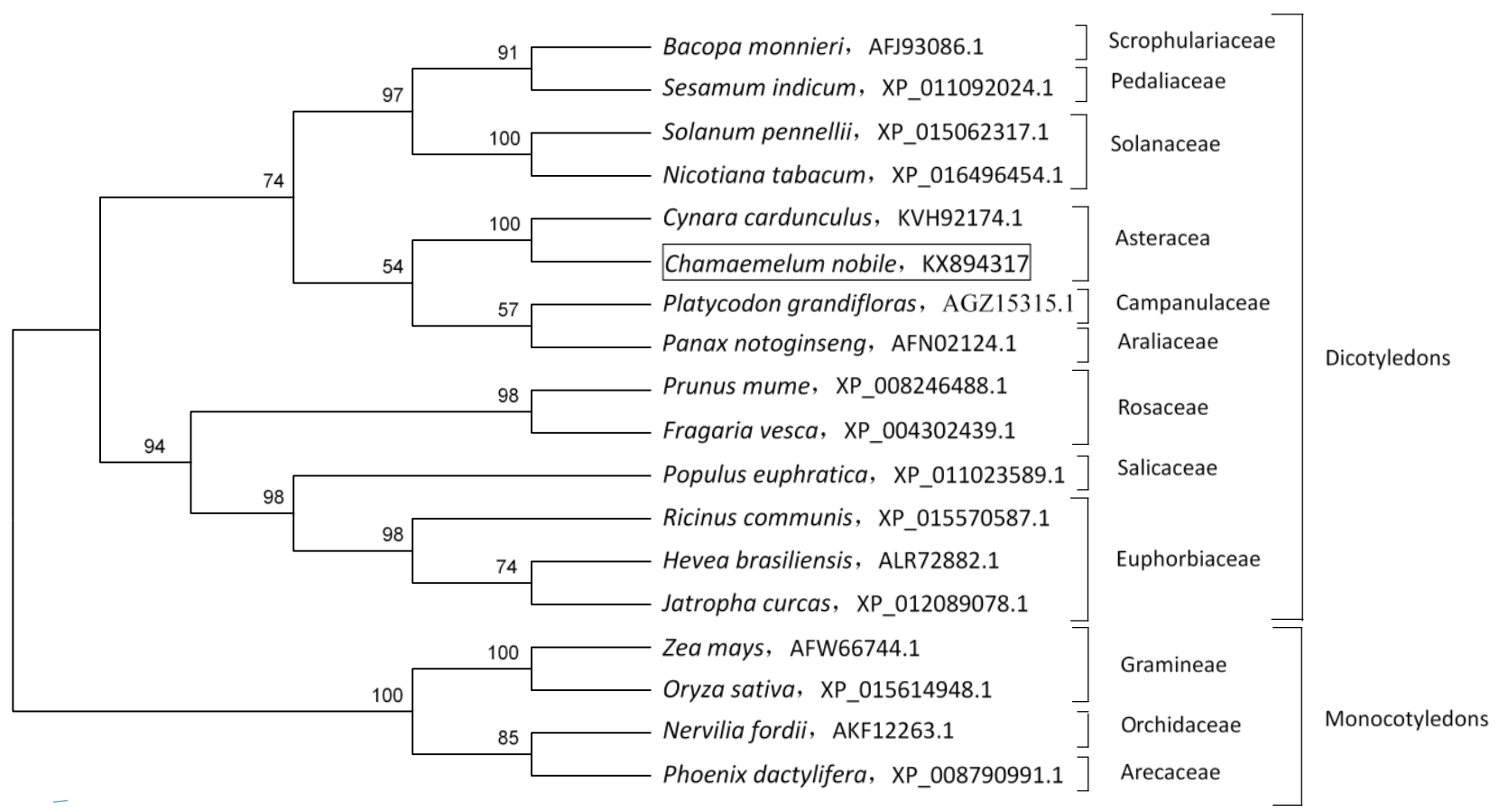

Fig. 3: Phylogenetic tree of MVK using Neighbor-Joining method. The number shown at each branch indicated the bootstrap values $(\%)$.

\section{Conclusion}

In this study, we have successfully isolated and cloned $C n M V K$ gene from $C$. nobile, the full-length cDNA of the gene is $1561 \mathrm{bp}$ with an ORF $1206 \mathrm{bp}$, encoding 402 amino acids. The multiple sequences alignment indicated that $C n M V K$ had high identity with other MVK genes isolated from other plants. The phylogenetic analysis demonstrated that $C n M V K$ keep a strong conservation during the molecular evolution and the evolutionary of plant MVK also keeps diversity. The study will provide evidence for the molecular function of $C n M V K$ and lay the foundation for the further application of $C n M V K$ in regulating the synthesis of terpenoid constituents. 


\section{Conflict of interest statement}

Authors declare that they have no conflict of interest.

\section{Acknowledgments}

This work was supported by the National Natural Science Foundation of China (31400603), International Science and Technology Cooperation Project of Hubei Province (2013BHE039 and 2013BHE039).

\section{References}

Alexandrov, N. N., Brover, V. V., Freidin, S., Troukhan, M. E., Tatarinova, T. V., Zhang, H. Y., Swaller, T. J., Lu, Y. P., Bouck, J., Flavell, R. B., Feldmann, K. A., 2009. Insights into corn genes derived from large-scale cDNA sequencing. Plant Mol. Biol. 69(1-2), 179-194.

Carnat, A., Carnat, A. P., Fraisse, D., Ricoux, L., Lamaison, J. L., 2004. The aromatic and polyphenolic composition of Roman chamomile tea. Fitoterapia. 75(1), 32-38.

Chu, X., Ding, L., 2003. Cloning, expression, and purification of His-tagged rat mevalonate kinase. Protein Expres. Purif. 27(1), 165-70.

Chu, X., Li, D., 2003. Expression, purification, and characterization of His20 mutants of rat mevalonate kinase. Protein Expres. Purif. 32(1), 75-82.

Dewick, P. M., 2002. The biosynthesis of C5-C25 terpenoid compounds. Nat. Prod. Rep. 19(2), 111-44.

Gundersen, S., Wibe, E., Funderud, S., Grzenlak-Puczyinska, I., Godal, T., 2015. Antibacterial, anti-swarming and antibiofilm formation activities of Chamaemelum nobile against Pseudomonas aeruginosa. Rev. Soc. Bras. Med. Tro. 48(4), 223-228.

Guo, X., Luo, H. M., Chen, S. L., 2012. Cloning and analysis of mevalonate kinase (PnMVKl) gene in Panax notoginseng. Acta Pharmaceut. Sinica. (8), 1092-1097.

Holstein, S., Hohl, R., 2004. Isoprenoids: Remarkable diversity of form and function. Lipids. 39(4), 293-309.

Huang, K. X., Scott, A. I., Bennett, G. N., 1999. Overexpression, purification and characterization of the thermostable mevalonate kinase from Methanococcus jannaschii. Protein Expres. Purif. 17(1), 33-40.

Huang, Q. L., He, R., Zhan, R. T., 2015. Identification and analysis of MVK gene in Nervilia fordii. Chin. J. Trop. Crops. 36(6), 1099-1104.

Larkin, M. A., Blackshields, G., Brown, N. P., Chenna, R., McGettigan, P. A., McWilliam, H., Valentin, F., Wallace, I. M., Wilm, A., Lopez, R., Thompson, J. D., Gibson, T. J., Higgins, D. G., 2007. Clustal W and Clustal X version 2.0. Bioinformatics. 23(21), 2947-2948.

Lemberkovics, E., Kery, A., Marczal, G., Simándi, B., Szöke, E., 1998. Phytochemical evaluation of essential oils, medicinal plants and their preparations. Acta Pharm. Hung. 68(3), 141-149.

Ma, Y., Yuan, L., Wu, B., Li, X., Chen, S., 2012. Genomewide identification and characterization of novel genes involved in terpenoid biosynthesis in Salvia miltiorrhiza. J. Exp. Bot. 63(7), 2809-2823.

Peña, D., Oca, N. M. D., Rojas, S., 2006. Anti-inflammatory and anti-diarrheic activity of Isocarpha cubana Blake. Pharmacology. 3, 744-749.

Riou, C., Tourte, Y., Lacroute, F., Karst, F., 1994. Isolation and characterization of a cDNA encoding Arabidopsis thaliana, mevalonate kinase by genetic complementation in yeast. Gene. 148(2), 293-297.

Simkin, A. J., Guirimand, G.., Papon, N., Papon, N., Courdavault, V., Thabet, I., Ginis, O., Bouzid, S., Giglioli-Guivarc'h, N., Clastre, M., 2011. Peroxisomal localisation of the final steps of the mevalonic acid pathway in plant. Planta. 234(5), 903-914.

Srivastava, J. K., Shankar, E., Gupta, S., 2010. Chamomile: A herbal medicine of the past with a bright future (Review). Mol. Med. Rep. 3(6), 895-901.

Wuyun, T. N., Wang, L., Ye, S. J., 2014. Identification and bioinformatics analysis of EuMK gene in Eucommia ulmoides. Nonwood For. Res. 32(1), 6-12.

\section{How to cite this article:}

Meng, X., Zhang, W., Xu, F., Yan, J., Liu, X., Liao, Y., Chang, J., 2016. Cloning and sequence analysis of mevalonate kinase gene (CnMVK) from Chamaemelum nobile. Int. J. Curr. Res. Biosci. Plant Biol. 3(11), 2328. doi: http://dx.doi.org/10.20546/ijcrbp.2016.311.004 